\title{
A new analytical thin-dusty-shell model to interpret interferometric data
}

\section{Application to the $S$ star $\pi^{1}$ Gruis}

\author{
P. Cruzalèbes and S. Sacuto

\begin{abstract}
Observatoire de la Côte d'Azur, Laboratoire Gemini, UMR-CNRS 6203, av. Copernic, 06130 Grasse, France
\end{abstract} \\ e-mail: pierre.cruzalebes@obs-azur.fr
}

Received 22 July 2005 / Accepted 6 February 2006

\section{ABSTRACT}

\begin{abstract}
High angular resolution techniques allow the determination of physical and geometrical parameters characterizing the circumstellar dusty environment of late-type stars. The resolution of the radiative transfer equation under the thin-shell approximation provides an analytical expression for the interferometric visibility profile, which can then be compared to simple ad-hoc geometrical models and to physical models based on radiative transfer. Using the DUSTY numerical code to calculate theoretical visibilities of the $\mathrm{S}$ star $\pi^{1} \mathrm{Gru}$, in agreement with spectrophotometric measurements from the near to the far infrared, we compared the validity of the output parameters found by the $\chi^{2}$ least-square analysis of the visibility, given with the thin-shell model and with a simple disk+ring geometrical model of the envelope. The results are discussed in terms of fitting the shape of the intensity profile and of the number of free parameters to be considered.
\end{abstract}

Key words. radiative transfer - methods: analytical - techniques: interferometric - circumstellar matter - stars: late-type infrared: stars

\section{Introduction}

Long-baseline large-aperture optical interferometers allow observation of faint astronomical sources at high spatial resolution. With its longest baseline of $200 \mathrm{~m}$, the ESO-VLTI on top of the Paranãl Mountain can reach the ultimate angular resolution of 2.3 mas at $2.2 \mu \mathrm{m}$. Thus, it should allow resolution of objects as small as $500 r_{\odot}$ located at $1 \mathrm{kpc}$ from Earth.

Among all the astrophysical sources that can be observed with the VLTI, late-type stars are privileged targets mainly thanks to their large angular scales and their high infrared brightness (Habing 1996). Under this same denomination, we include the AGB cool stars, with effective temperatures ranging from 2500 to $3500 \mathrm{~K}$, with luminosities from $10^{3}$ to $10^{4} L_{\odot}$, and with radii from $10^{2}$ to $10^{3} r_{\odot}$. With mass-loss rates as high as $10^{-4} M_{\odot} \mathrm{y}^{-1}$, the observation of their close circumstellar environment with optical interferometers gives information on the formation, on the nature, and on the evolution of the dust created in the expanding shell. Warm molecular layers recently revealed by narrow-band interferometric measurements in the upper atmosphere of some late-type stars (Perrin et al. 2004; Ohnaka et al. 2005; Perrin et al. 2005) will not be considered in our study, which concentrates on further circumstellar dusty shells mainly responsible for the radiation emitted in the continuum at infrared wavelengths.

Dust grains are usually supposed to form at such a distance from the stellar surface that the total energy density is equal to that of a black body, whose effective temperature is called the grain condensation temperature $T_{\text {cond }}$ (Papoular \& Pégourié 1986). As confirmed by ISI-Mount Wilson interferometric measurements (Danchi et al. 1994), grain condensation occurs between 2 and $10 r_{\star}$, depending on the central star temperature and on the chemical nature of the grains (Zuckerman 1980). In the most commonly used models, the circumstellar dusty envelope contains a dissociation zone of the grain precursor molecules, forming a spherical cavity around the central star that defines the shell's inner boundary (Rowan-Robinson 1980). Within this cavity, the temperature of the circumstellar medium warmed by the stellar radiation remains higher than the grain sublimation temperature $T_{\text {sub }}$ (generally assumed to be equal to $\left.T_{\text {cond }}\right)$, so the dust grains cannot survive. At thermodynamic equilibrium, the radius of the shell's inner boundary is given by

$\frac{r_{\text {in }}}{r_{\star}}=\alpha_{0}\left(\frac{T_{\star}}{T_{\text {sub }}}\right)^{2}$,

where $T_{\star}$ and $r_{\star}$ are the stellar temperature and its radius, respectively, and $\alpha_{0}$ a dimensionless parameter. Depending mainly on the optical properties of the grains, this radius is almost independent of $T_{\star}$ and $T_{\text {sub }}$ and weakly dependent on the overall monochromatic optical depth of the envelope (Ivezić \& Elitzur 1996). This dependency needs the analytical treatment of the radiative transfer in the dusty shell, which can be successfully undertaken only in some simple cases, such us under the thindusty-shell condition, which is one of the main assumptions of our study.

The determination of the intensity at the inner boundary also needs the resolution of the radiative transfer equation in the dusty shell. It was demonstrated that, when the shell is optically thin at all wavelengths, a very sharp maximum of intensity appears at the inner boundary, creating a discontinuity in the slope of the intensity profile and forming a bright annulus around the central star (Rowan-Robinson 1980; Lefèvre et al. 1982). 
The angular distribution of the radiation emitted by the shell can be separated into 2 distinct directions, called the "core" and the "shell" directions (Mitchell \& Robinson 1978). In the core direction, the intensity increases until a maximum value reached in the direction of the shell's inner boundary, which is a simple consequence of the thickness of dust, maximum in the direction of the inner radius. In the shell direction, the emitted radiation falls off from the shell's inner boundary to the outer boundary. When the dust is optically thick, the solution is not straightforward, so the problem must be solved numerically (Ivezić \& Elitzur 1997). The intensity peak at the inner boundary disappears and the slope tends to follow a featureless bell-shaped curve starting from the central star and rapidly decreasing towards the outer limit of the shell (Ivezić \& Elitzur 1996; Mitchell \& Robinson 1978; Rowan-Robinson 1980). At interferometric resolutions, the detection of the shell's inner boundary can be achieved, as already done with ISI (Danchi et al. 1994). This provides a powerful tool for fixing the distance at which the dust grain nuclei can condensate and grow and estimates the dust mass-loss rate.

In the present paper, we propose to use a new thin-dustyshell model based on analytical expression of the optical depth deduced from the formal resolution of the radiative transfer equation, formally depending on the inner radius and providing quicker automatic estimations of the physical parameters and of their confidence intervals. Our model gives intensity and visibility profiles close to those produced by the publicly available DUSTY code, widely used in the community. This comparison is made in the special case of the observation of the $S$ star $\pi^{1}$ Gru. To deduce the physicochemical parameters of its dusty shell, real infrared spectrophotometric data are fitted thanks to DUSTY. The output visibility function produced by DUSTY with this set of parameters is then compared to the visibility profile calculated with our analytical thin-dusty-shell model. The results are discussed in terms of an estimation of the inner-boundary radius. We particularly insist on the influence of the fitting quality and of the free parameter number on the reliability of the parameter estimation.

\section{Thin-dusty-shell analytical model}

\subsection{Approximated solution of the radiative transfer equation}

Models generally produce sets of output data that can be directly compared to the measurements, such as the spectral energy distribution of the source, its output brightness, and visibility. In order to fit the measurements with a model and determine the physical parameters used as input parameters of that model, the $\chi^{2}$ least-square analysis is widely used, which tries to minimize the sum of the square of the deviations between the data and the model. In general, numerical models based on the radiative transfer, which are used to interpret the data, do not provide analytical expressions of the visibility function. For this reason, we propose a new analytical model that explicitly depends on a few physical and geometrical parameters including the inner boundary radius.

When assuming that the dusty medium is composed of single-type dust grains considered as black bodies of effective temperature $T_{\mathrm{g}}$, ignoring the scattered radiation as long as the grain size remains much smaller than the wavelength (Marengo 2000), and neglecting the angular dependency of the optical depth contained in the central radiation term, the local monochromatic intensity at a point $P$ (inside the dusty shell) located at a distance $r$ to the center $C$ along an optical path $(\mathcal{P})$ forming an angle $\theta$ with the radial direction (see Fig. 1), formal

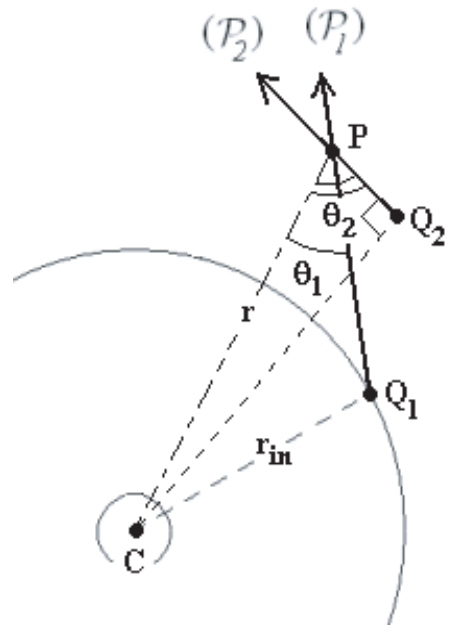

Fig. 1. Schematic illustration of the geometry used in the present study. Here, $r$ is the radial distance of the point $P$ (located in the dust shell) from the center of symmetry $C$, while $\theta_{1}$ and $\theta_{2}$ define the directions of the optical paths $\left(\mathcal{P}_{1}\right)$ and $\left(\mathcal{P}_{2}\right)$. The largest circle defines the inner shell cavity of radius $r_{\text {in }}$, while the smallest circle around the center $C$ defines the stellar disk of radius $r_{\star}$. The optical path $\left(\mathcal{P}_{1}\right)$ crosses the inner cavity (core direction), so that the radial distance of the closest approach to the center $\left(Q_{1}\right)$ is equal to $r_{\text {in }}$. The optical path $\left(\mathcal{P}_{2}\right)$ never crosses the inner cavity (shell direction), so that the radial distance of the closest approach to the center $\left(Q_{2}\right)$ is now equal to $r \sin \theta_{2}$.

solution of the radiative transfer equation in presence of a central radiation source, can be given by

$$
\begin{aligned}
I_{\lambda}(r ; \theta) \approx & I_{\star \lambda} \exp \left(-\tau_{\lambda}(r ; 0)\right) \Theta\left(r_{\star}-r \sin \theta\right) \Theta(\cos \theta) \\
& +B_{\lambda}\left(T_{\mathrm{g}}\right)\left[1-\exp \left(-\tau_{\lambda}(r ; \theta)\right)\right]
\end{aligned}
$$

where $\tau_{\lambda}$ is the local optical depth at distance $r$ along $(\mathcal{P})$, defined by $\mathrm{d} \tau_{\lambda}=\kappa_{\lambda} \mathrm{d} s\left(\kappa_{\lambda}\right.$ is the extinction coefficient and $\mathrm{d} s$ the path-length element), $\Theta$ the Heaviside unit step function $\left(\Theta(x)=1\right.$ if $x \geqslant 0$, zero otherwise), $r_{\star}$ the radius of the central star, $I_{\star \lambda}$ the intensity of the central star of effective temperature $T_{\star}$, and $B_{\lambda}\left(T_{\mathrm{g}}\right)$ is the Planck distribution with the grain temperature $T_{\mathrm{g}}$.

If the dust has a spherically symmetric distribution around the central star, the local optical depth from the closest approach to the center along $(\mathcal{P})$ (Ivezić \& Elitzur 1997) is given by

$\tau_{\lambda}(r ; \theta)=Q_{\lambda} \sigma_{\mathrm{g}} \int_{(\mathcal{P})} n\left(\sqrt{s^{2}+r^{2} \sin ^{2} \theta}\right) \mathrm{d} s$,

where $Q_{\lambda}=\kappa_{\lambda} /\left(n \sigma_{\mathrm{g}}\right)$ is the optical efficiency of the particle material, $n$ the radial number density of the grains, and $\sigma_{\mathrm{g}}$ their geometrical cross section.

If we suppose a dustless spherical cavity of radius $r_{\text {in }}$ around the central source $\left(r_{\text {in }}>r_{\star}\right)$, two different situations can be identified according to whether the optical path crosses the dusty shell's inner boundary or not. Using $r \cos \theta-s$ instead of $s$ in Eq. (3), the general expression of the optical depth becomes

$\tau_{\lambda}(r ; \theta)=Q_{\lambda} \sigma_{\mathrm{g}} \int_{0}^{l(r ; \theta)} n\left(\sqrt{(r \cos \theta-s)^{2}+r^{2} \sin ^{2} \theta}\right) \mathrm{d} s$,

where $l(r ; \theta)$ is the geometrical thickness along $(\mathcal{P})$, equal to $r \cos \theta-\sqrt{r_{\text {in }}^{2}-r^{2} \sin ^{2} \theta}$ if $0 \leqslant r \sin \theta \leqslant r_{\text {in }}$ (the "core" direction), equal to $r \cos \theta$ if $r \sin \theta \geqslant r_{\text {in }}$ (the "shell" direction). These two 


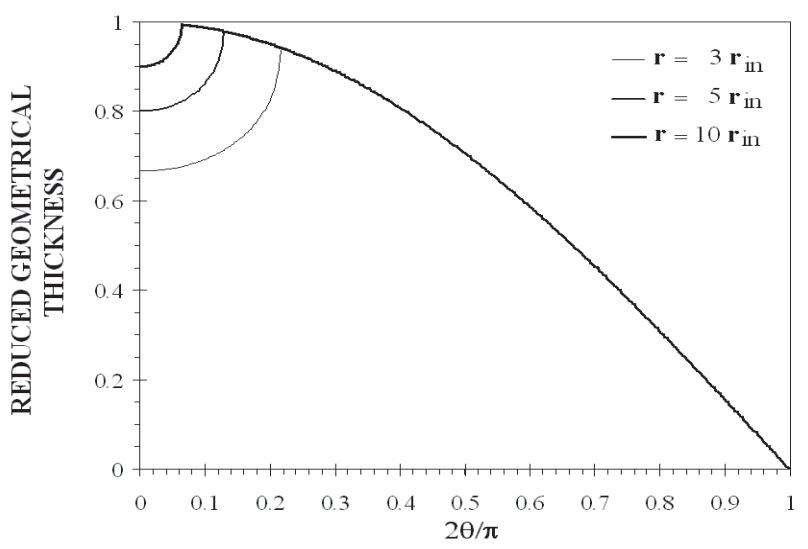

Fig. 2. Angular variation in the reduced geometrical thickness $l(r ; \theta) / r$ of Eq. (5) for different values of the radial distance $r$ (in $r_{\text {in }}$ units).

cases, illustrated in Fig. 1, can be gathered in a single expression using the $\Theta$ function introduced in Eq. (2)

$l(r ; \theta)=r \cos \theta-\Theta\left(r_{\text {in }}-r \sin \theta\right) \sqrt{r_{\text {in }}^{2}-r^{2} \sin ^{2} \theta}$.

Figure 2 shows the angular dependency of the reduced geometrical thickness $l(r ; \theta) / r$ of Eq. (5) for different values of the radial distance $r$, expressed in $r_{\text {in }}$ units. Starting from $\theta=0$ where it is equal to $1-r_{\text {in }} / r$, the reduced geometrical thickness increases rapidly in the core region up to a maximum value equal to $\sqrt{1-r_{\text {in }}^{2} / r^{2}}$ at $\theta=\theta_{\text {in }}=\arcsin \left(r_{\text {in }} / r\right)$. Then, it slowly decreases in the shell region down to zero at $\theta=\pi / 2$.

If we suppose that $n(r)$ decreases from the inner boundary radius as a power law (van der Veen et al. 1995), i.e. $n(r)=$ $n_{\text {in }}\left(r_{\text {in }} / r\right)^{p}$, where $n_{\text {in }}=n\left(r_{\text {in }}\right)(p \geqslant 0)$, Eq. (4) becomes

$\tau_{\lambda}(r ; \theta)=Q_{\lambda} \sigma_{\mathrm{g}} n_{\text {in }} r_{\text {in }}^{p} \int_{0}^{l(r ; \theta)} \frac{\mathrm{d} s}{\left[(r \cos \theta-s)^{2}+r^{2} \sin ^{2} \theta\right]^{\frac{p}{2}}}$.

The formal resolution of the integral contained in this equation leads to the approximated expression of the local optical depth if $l(r ; \theta) \ll r$ (geometrically thin-dusty-shell approximation)

$\tau_{\lambda}(r ; \theta) \approx \kappa_{\lambda}^{\text {in }}\left(\frac{r_{\text {in }}}{r}\right)^{p} l(r ; \theta) \approx Q_{\lambda} \sigma_{\mathrm{g}} n(r) l(r ; \theta)$,

where $\kappa_{\lambda}^{\text {in }}=Q_{\lambda} \sigma_{\mathrm{g}} n_{\text {in }}$ is the extinction coefficient at the inner boundary.

The general expression of the geometrical thickness of Eq. (5) allows us to get the analytical expression of the local optical depth according to the grain number density. Figure 3 shows the angular variation of the reduced optical depth $\tau_{\lambda}(r ; \theta) /\left(\kappa_{\lambda}^{\text {in }} r\right)$ using the exact solutions of the integration of Eq. (6) for different values of $p$ with $r=5 r_{\text {in }}$, while Fig. 4 shows the angular variation of the reduced optical depth for different values of the radial distance $r$ (in $r_{\text {in }}$ units), with $p=2$.

\subsection{Analytical expression of the intensity profile}

To find the expression of the total emergent intensity, we need to distinguish between the various dominant sources of radiation according to the direction of the line of sight. Figure 5 shows the geometry used. The intensity $I_{\lambda}(\varepsilon)$ received by the observer located at $O$, at a distance $D$ from $C$ (in $\mathrm{W} \mathrm{m}^{-2} \mu \mathrm{m}^{-1} \mathrm{sr}^{-1}$ ), is equal to the emergent intensity $I_{\lambda}\left(r_{\text {out }} ; \theta\right)$ coming from the point $P$ located at the inner boundary of the dusty shell of radius $r_{\text {out }}$.

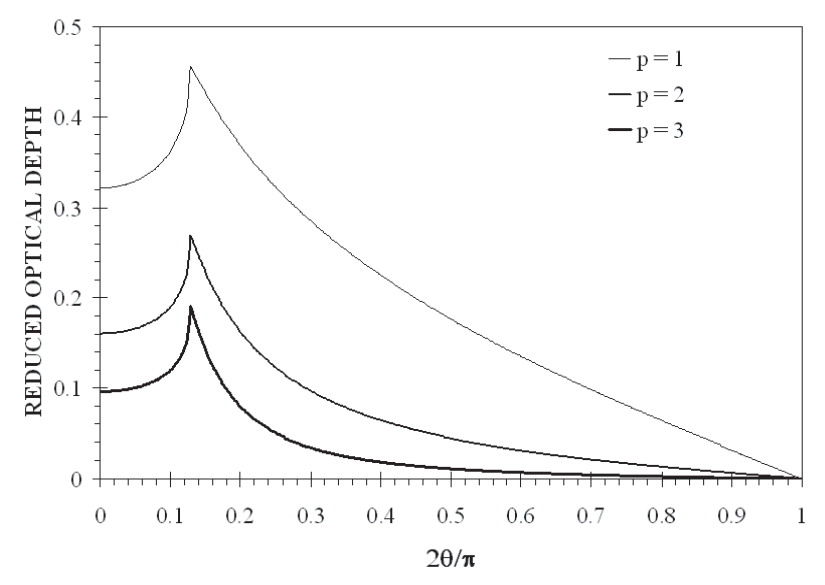

Fig. 3. Angular variation of the reduced optical depth $\tau_{\lambda}(r ; \theta) /\left(\kappa_{\lambda}^{\text {in }} r\right)$ using the exact solutions of the integration of Eq. (6) for different values of the power $p$ of the radial number density, with $r=5 r_{\text {in }}$.

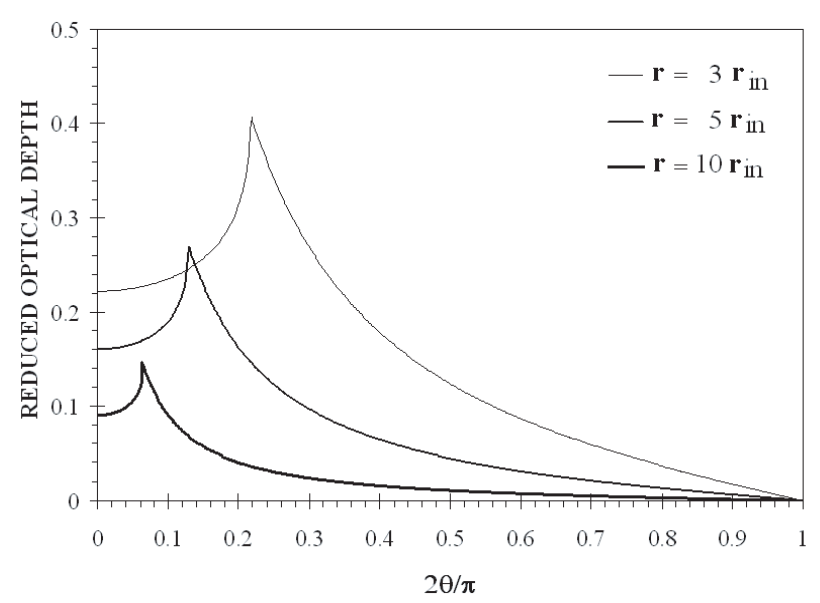

Fig. 4. Angular variation of the reduced optical depth $\tau_{\lambda}(r ; \theta) /\left(\kappa_{\lambda}^{\text {in }} r\right)$ using the exact solutions of the integration of Eq. (6) with $p=2$, for different values of the radial distance $r$ (in $r_{\text {in }}$ units).

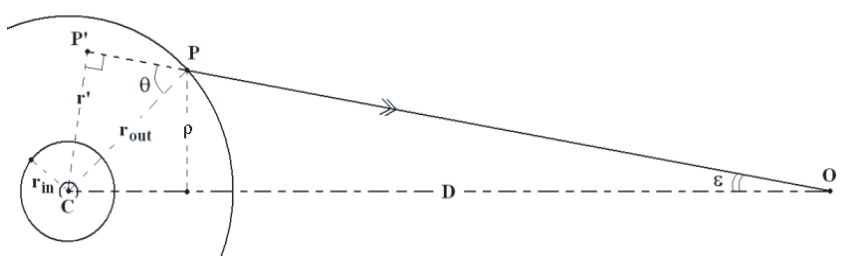

Fig. 5. Geometry used for determining the emergent intensity from the point $P$ located at the outer boundary of radius $r_{\text {out }}$ and received under the angle $\varepsilon$ by the observer located at $O$, at a distance $D$ from the center $C$ of the central source. The $P^{\prime}$ point, the closest approach to the center, is located at $r^{\prime} ; \rho$ is the impact parameter, and $r_{\text {in }}$ the inner boundary radius.

The stellar radiation, considered as being created by a blackbody of temperature $T_{\star}$ for simplification, is only present in the central direction $\left(0 \leqslant \varepsilon \leqslant \varepsilon_{\star} \approx r_{\star} / D\right)$. Since the stellar size is small compared to the inner shell radius, the angular variation of $\tau_{\lambda}\left(r_{\text {out }} ; \theta\right)$ can be neglected in these directions, so that the stellar intensity distribution can be assumed as quasi constant

$I_{\lambda}^{\star}(\varepsilon) \approx B_{\lambda}\left(T_{\star}\right) \Pi\left(\frac{\varepsilon}{2 \varepsilon_{\star}}\right)$, 
where $\Pi$ is the uniform disk distribution $(\Pi(x)=1$ if $-1 / 2 \leqslant$ $x \leqslant 1 / 2$, zero otherwise). Under the thin limit condition (1 $\exp \left(-\tau_{\lambda}\right) \approx \tau_{\lambda}$ for any $\left.\lambda\right)$, the intensity produced by the dusty envelope in all directions is

$I_{\lambda}^{\text {env }}(\varepsilon) \approx B_{\lambda}\left(T_{\mathrm{g}}\right) \tau_{\lambda}\left(r_{\text {out }} ; \theta\right)$.

Using $\varepsilon \approx\left(r_{\text {out }} / D\right) \sin \theta$ (see Fig. 5), the total geometrical thickness of Eq. (5) becomes

$l\left(r_{\text {out }} ; \varepsilon\right)=D\left[\sqrt{\varepsilon_{\text {out }}^{2}-\varepsilon^{2}}-\Theta\left(\varepsilon_{\text {in }}-\varepsilon\right) \sqrt{\varepsilon_{\text {in }}^{2}-\varepsilon^{2}}\right]$,

where $\varepsilon_{\text {in }} \approx r_{\text {in }} / D$ and $\varepsilon_{\text {out }} \approx r_{\text {out }} / D$. In the core direction $(0 \leqslant$ $\left.\varepsilon \leqslant \varepsilon_{\text {in }}\right)$, the output radiation produced by the dusty envelope is dominated by the grains located very close to the inner boundary, so that $T_{\mathrm{g}}=T_{\text {in }}$. In the shell direction $\left(\varepsilon_{\text {in }} \leqslant \varepsilon \leqslant \varepsilon_{\text {out }}\right)$, we can consider, as a first approximation, that the main contributors to the emergent intensity are the dust grains located at the closest approach $P^{\prime}$ to the center (see Fig. 5), located at a distance $r^{\prime}=$ $r_{\text {out }} \sin \theta$ from $C$, which are at the same time the hottest and the most numerous grains along the optical path.

To get an approximated expression of the grain temperature distribution at $P^{\prime}$, we must use the radiative equilibrium condition at radial distance $r^{\prime}$, obtained by angular integration and by symmetry, according to

$\int_{0}^{\infty} \kappa_{\lambda} J_{\lambda}\left(r^{\prime}\right) \mathrm{d} \lambda=\int_{0}^{\infty} \kappa_{\lambda} B_{\lambda}\left(T_{\mathrm{g}}\right) \mathrm{d} \lambda$

where $J_{\lambda}$ is the angle-averaged mean intensity defined by $J_{\lambda}\left(r^{\prime}\right)=(1 / 2) \int_{-1}^{1} I_{\lambda}\left(r^{\prime} ; \mu\right) \mathrm{d} \mu(\mu=\cos \theta)$.

Thanks to Eq. (2) one gets

$$
\begin{aligned}
J_{\lambda}\left(r^{\prime}\right) \approx & \frac{1}{2} \int_{\mu_{\star}}^{1} B_{\lambda}\left(T_{\star}\right) \exp \left(-\tau_{\lambda}\left(r^{\prime} ; \mu=1\right)\right) \mathrm{d} \mu \\
& +B_{\lambda}\left(T_{\mathrm{g}}\right)\left[\frac{1}{2} \int_{-1}^{1}\left[1-\exp \left(-\tau_{\lambda}\left(r^{\prime} ; \mu\right)\right)\right] \mathrm{d} \mu\right],
\end{aligned}
$$

where $\mu_{\star}=\cos \theta_{\star}=\sqrt{1-r_{\star}^{2} / r^{\prime 2}}$, so that the radiative equilibrium condition becomes

$$
\begin{aligned}
\left(1-\mu_{\star}\right) & \int_{0}^{\infty} \kappa_{\lambda} B_{\lambda}\left(T_{\star}\right) \exp \left(-\tau_{\lambda}\left(r^{\prime} ; 1\right)\right) \mathrm{d} \lambda \approx \\
& \int_{0}^{\infty} \kappa_{\lambda} B_{\lambda}\left(T_{\mathrm{g}}\right)\left[\int_{-1}^{1} \exp \left(-\tau_{\lambda}\left(r^{\prime} ; \mu\right)\right) \mathrm{d} \mu\right] \mathrm{d} \lambda .
\end{aligned}
$$

Using the peak shape of the Planck distribution, i.e. $\int_{0}^{\infty} f_{\lambda} B_{\lambda}(T) \mathrm{d} \lambda \approx f_{\lambda_{\max }}\left(\sigma T^{4} / \pi\right)$, where $\lambda_{\max }$ is given by the Wien displacement law $\lambda_{\max } T=$ const. the radiative equilibrium at the radial distance $r^{\prime}$ becomes

$$
\begin{array}{r}
\left(1-\mu_{\star}\right) \kappa_{\lambda_{\star}} T_{\star}^{4} \exp \left(-\tau_{\lambda_{\star}}\left(r^{\prime} ; 1\right)\right) \approx \\
\kappa_{\lambda_{\mathrm{g}}} T_{\mathrm{g}}^{4} \int_{-1}^{1} \exp \left(-\tau_{\lambda_{\mathrm{g}}}\left(r^{\prime} ; \mu\right)\right) \mathrm{d} \mu,
\end{array}
$$

where $\lambda_{\star}$ and $\lambda_{\mathrm{g}}$ are given by $\lambda_{\star} T_{\star}=\lambda_{\mathrm{g}} T_{\mathrm{g}}$. If the dusty shell is optically thin $\left(\tau_{\lambda} \approx 0\right.$ for any $\lambda$ ) and assuming that $r_{\star} \ll r^{\prime}$, the radiative equilibrium equation gives the grain temperature distribution

$$
\left(\frac{T_{\mathrm{g}}}{T_{\star}}\right)^{4} \approx \frac{1}{4} \frac{\kappa_{\lambda_{\star}}}{\kappa_{\lambda_{\mathrm{g}}}}\left(\frac{r_{\star}}{r^{\prime}}\right)^{2} .
$$

In particular, $T_{\mathrm{g}}=T_{\text {in }}$ if $r^{\prime}=r_{\mathrm{in}}$, so that

$$
\left(\frac{T_{\mathrm{in}}}{T_{\star}}\right)^{4} \approx \frac{1}{4} \frac{\kappa_{\lambda_{\star}}}{\kappa_{\lambda_{\mathrm{in}}}}\left(\frac{r_{\star}}{r_{\mathrm{in}}}\right)^{2}
$$

Dividing Eq. (15) by Eq. (16), we obtain

$$
\left(\frac{T_{\mathrm{g}}}{T_{\mathrm{in}}}\right)^{4} \approx \frac{\kappa_{\lambda_{\mathrm{in}}}}{\kappa_{\lambda_{\mathrm{g}}}}\left(\frac{r_{\mathrm{in}}}{r^{\prime}}\right)^{2}
$$

If $\lambda_{\text {in }}$ and $\lambda_{\mathrm{g}}$ are in the regime where $Q_{\lambda} \propto \lambda^{-\beta},(\beta \geqslant 0)$ (Sopka et al. 1985), and using the Wien displacement law $\left(\lambda_{\mathrm{g}} T_{\mathrm{g}}=\right.$ $\left.\lambda_{\text {in }} T_{\text {in }}\right)$, the distribution of the grain temperature becomes

$\frac{T_{\mathrm{g}}\left(r^{\prime}\right)}{T_{\mathrm{in}}} \approx\left(\frac{r_{\mathrm{in}}}{r^{\prime}}\right)^{\frac{2}{\beta+4}}$

so that by introducing $\varepsilon / \varepsilon_{\text {in }} \approx r^{\prime} / r_{\text {in }}$ we get

$T_{\mathrm{g}}(\varepsilon) \approx T_{\text {in }}\left(\frac{\varepsilon}{\varepsilon_{\text {in }}}\right)^{-\frac{2}{\beta+4}}$

where the value of $\beta$ is generally taken between 1 and 2 for silicated and carbonaceous dust grains (Marengo et al. 1997).

Finally, using Eqs. (7), (9), and (10), we get the following general expressions of the envelope intensity for small optical depths:

1. In the core direction $\left(0 \leqslant \varepsilon \leqslant \varepsilon_{\text {in }}\right)$ :

$$
\begin{aligned}
I_{\lambda}^{\text {core }}(\varepsilon) \approx & B_{\lambda}\left(T_{\text {in }}\right) \kappa_{\lambda}^{\text {in }}\left(\frac{\varepsilon_{\text {in }}}{\varepsilon_{\text {out }}}\right)^{p} D \\
& \times\left[\sqrt{\varepsilon_{\text {out }}^{2}-\varepsilon^{2}}-\sqrt{\varepsilon_{\text {in }}^{2}-\varepsilon^{2}}\right] .
\end{aligned}
$$

2. In the shell direction $\left(\varepsilon_{\text {in }} \leqslant \varepsilon \leqslant \varepsilon_{\text {out }}\right)$ :

$$
I_{\lambda}^{\text {shell }}(\varepsilon) \approx B_{\lambda}\left(T_{\mathrm{g}}(\varepsilon)\right) \kappa_{\lambda}^{\text {in }}\left(\frac{\varepsilon_{\text {in }}}{\varepsilon_{\text {out }}}\right)^{p} D \sqrt{\varepsilon_{\text {out }}^{2}-\varepsilon^{2}},
$$

where $T_{\mathrm{g}}(\varepsilon) \approx T_{\text {in }}\left(\varepsilon / \varepsilon_{\text {in }}\right)^{-2 /(\beta+4)}$.

At $\varepsilon=\varepsilon_{\text {in }}$, the continuity of the intensity profile is insured, for we have

$$
\begin{aligned}
I_{\lambda}^{\text {core }}\left(\varepsilon_{\text {in }}\right) & =I_{\lambda}^{\text {shell }}\left(\varepsilon_{\text {in }}\right)=I_{\lambda}^{\text {in }} \\
& \approx B_{\lambda}\left(T_{\text {in }}\right) \kappa_{\lambda}^{\text {in }}\left(\frac{\varepsilon_{\text {in }}}{\varepsilon_{\text {out }}}\right)^{p} D \sqrt{\varepsilon_{\text {out }}^{2}-\varepsilon_{\text {in }}^{2}}
\end{aligned}
$$

These expressions are in good agreement with Ridgway's analytical face-on ring model (Ridgway et al. 1986) if we assume $T_{\mathrm{g}}=T_{\text {in }}$ in our model.

Figure 6 shows the variation of the reduced incident intensity $I_{\lambda}(\varepsilon) /\left(B_{\lambda}\left(T_{\text {in }}\right) \kappa_{\lambda}^{\text {in }} \varepsilon_{\text {in }} D\right)$ versus the reduced impact parameter $\varepsilon / \varepsilon_{\text {in }}$ for different values of $p$ with $r_{\text {out }}=5 r_{\text {in }}, \beta=1$, and $T_{\text {in }}=1000 \mathrm{~K}$, while Figs. 7-9 show the variation in the reduced incident intensity versus the reduced impact parameter for different values of, respectively,

- $r_{\text {out }}$ (in $r_{\text {in }}$ units) with $p=2, \beta=1$, and $T_{\text {in }}=1000 \mathrm{~K}$;

$-\beta$, with $p=2, r_{\text {out }}=5 r_{\text {in }}$, and $T_{\text {in }}=1000 \mathrm{~K}$;

$-T_{\text {in }}$, with $p=2, r_{\text {out }}=5 r_{\text {in }}$, and $\beta=1$. 


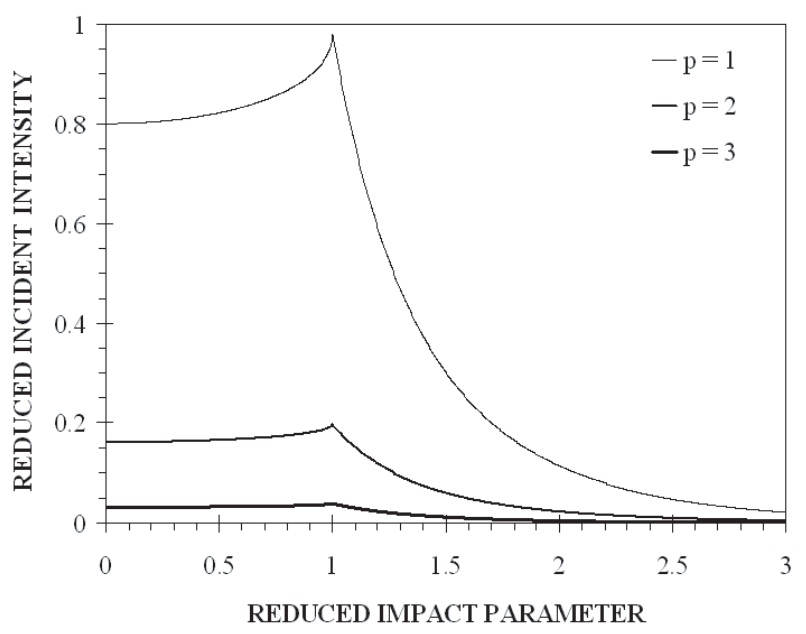

Fig. 6. Variation in the reduced incident intensity $I_{\lambda}(\varepsilon) /\left(B_{\lambda}\left(T_{\text {in }}\right) \kappa_{\lambda}^{\text {in }} \varepsilon_{\text {in }} D\right)$ versus the reduced impact parameter $\varepsilon / \varepsilon_{\text {in }}$ for different values of the power $p$ with $r_{\text {out }}=5 r_{\text {in }}, \beta=1$, and $T_{\text {in }}=1000 \mathrm{~K}$.

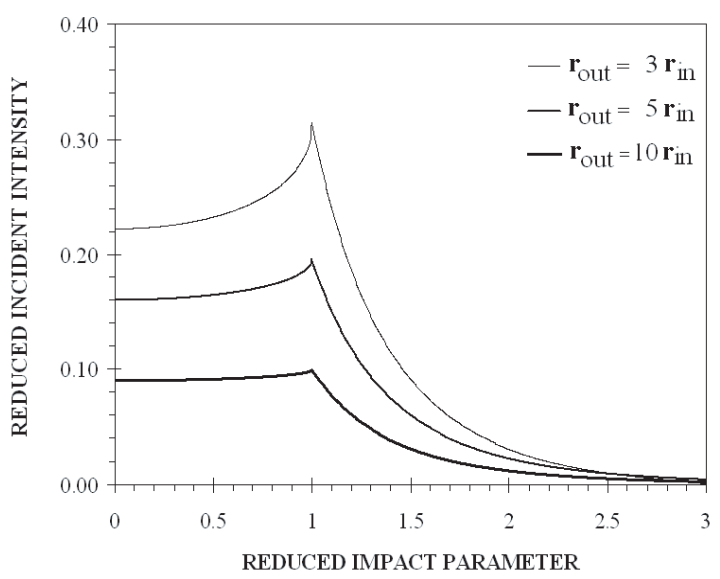

Fig. 7. Variation in the reduced incident intensity $I_{\lambda}(\varepsilon) /\left(B_{\lambda}\left(T_{\text {in }}\right) \kappa_{\lambda}^{\text {in }} \varepsilon_{\text {in }} D\right)$ versus the reduced impact parameter $\varepsilon / \varepsilon_{\text {in }}$ for different values of $r_{\text {out }}$ (in $r_{\text {in }}$ units) with $p=2, \beta=1$, and $T_{\text {in }}=1000 \mathrm{~K}$.

\subsection{Analytical expression of the visibility profile}

In the case of spherical symmetry, where $I_{\lambda}$ depends only on the radial angular distance $\varepsilon$, the monochromatic visibility function $V_{\lambda}$ measured by an interferometer is linked to the source monochromatic brightness angular distribution $I_{\lambda}(\varepsilon)$ thanks to the normalized Hankel transform according to

$V_{\lambda}\left(\frac{b_{i j}}{\lambda}\right)=\left|\frac{\breve{I}_{\lambda}\left(\frac{b_{i j}}{\lambda}\right)}{\breve{I}_{\lambda}(0)}\right|$,

where $b_{i j}$ is the modulus of the projected baseline (on the plane of the sky) $\boldsymbol{b}_{i j}$ formed by the pair of apertures $(i ; j)$, and where

$\breve{I}_{\lambda}(f)=\int_{0}^{\infty} 2 \pi \varepsilon I_{\lambda}(\varepsilon) J_{0}(2 \pi f \varepsilon) \mathrm{d} \varepsilon$

is the Hankel transform of $I_{\lambda}$ at the spatial frequency $f$ ( $J_{0}$ being the zeroth order Bessel function of the first kind).

In our study, the total intensity profile results from the central star, considered as a uniform disk, and from the circumstellar envelope according to $I_{\lambda}(\varepsilon)=I_{\lambda}^{\star}(\varepsilon)+I_{\lambda}^{\text {env }}(\varepsilon)$, where $I_{\lambda}^{\star}$ is given by Eq. (8), while $I_{\lambda}^{\text {env }}$ is given by Eqs. (20) or (21) according to the direction of the line of sight.

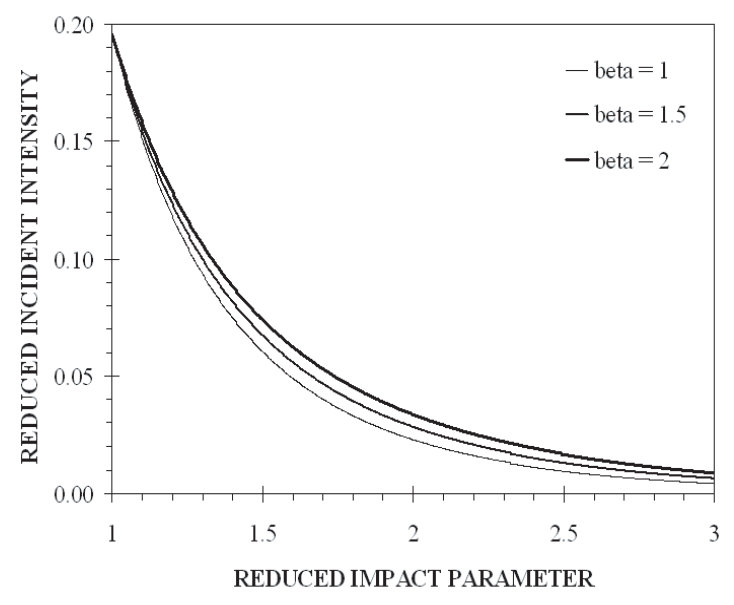

Fig. 8. Variation in the reduced incident intensity $I_{\lambda}^{\text {shell }}(\varepsilon) /\left(B_{\lambda}\left(T_{\text {in }}\right) \kappa_{\lambda}^{\text {in }} \varepsilon_{\text {in }} D\right)$ versus the reduced impact parameter $\varepsilon / \varepsilon_{\text {in }}$ for different values of $\beta$, with $p=2, r_{\text {out }}=5 r_{\text {in }}$, and $T_{\text {in }}=1000 \mathrm{~K}$.

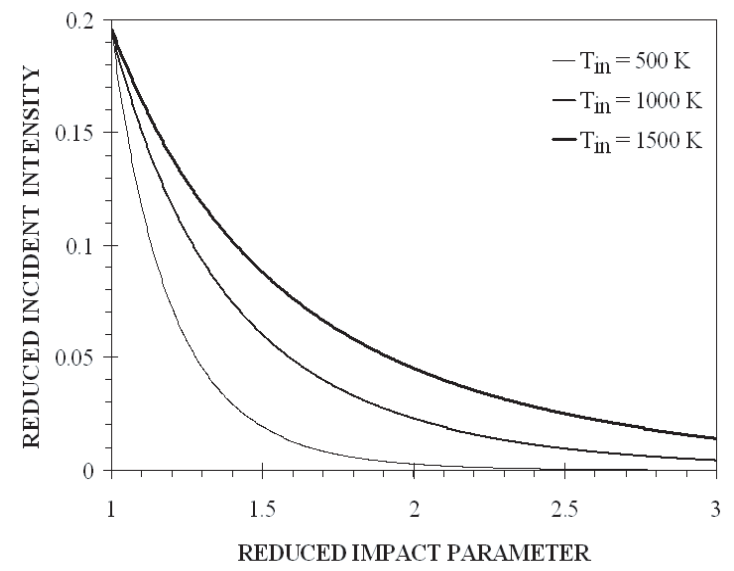

Fig. 9. Variation in shell reduced incident intensity $I_{\lambda}^{\text {shell }}(\varepsilon) /\left(B_{\lambda}\left(T_{\text {in }}\right) \kappa_{\lambda}^{\text {in }} \varepsilon_{\text {in }} D\right)$ versus the reduced impact parameter $\varepsilon / \varepsilon_{\text {in }}$ for different values of the inner boundary temperature $T_{\text {in }}$, with $p=2, r_{\text {out }}=5 r_{\text {in }}$, and $\beta=1$.

Although some canonical Hankel transforms can be found in the literature (Bracewell 1999) along with some simple integrals of Bessel functions (Abramowitz \& Stegun 1972), which can give the explicit dependency of the visibility function according to the geometrical parameters that describe the intensity profile, more complex analytical expressions of the radial intensity profiles generally have no simple analytical expression of their Hankel transform. In our study, where the intensity profile follows three different laws in the central, in the core, and in the shell directions, we can use the well-known general trapezoidal rule to evaluate the Bessel integrals (Barakat et al. 1998), so that the visibility profile can be estimated by ( $J_{1}$ being the first order Bessel function of the first kind)

$V_{\lambda}(f)=\left|\frac{\breve{I}_{\lambda}^{\star}(f)+\breve{I}_{\lambda}^{\text {core }}(f)+\breve{I}_{\lambda}^{\text {shell }}(f)}{\breve{I}_{\lambda}^{\star}(0)+\breve{I}_{\lambda}^{\text {core }}(0)+\breve{I}_{\lambda}^{\text {shell }}(0)}\right|$,

where

$$
\begin{aligned}
& -\breve{I}_{\lambda}^{\star}(f)=\varepsilon_{\star} I_{\lambda}^{\star} J_{1}\left(2 \pi f \varepsilon_{\star}\right) / f ; \\
& \text { - } \breve{I}_{\lambda}^{\text {core }}(f) \approx 2 \pi h_{\text {core }} \sum_{n=1}^{N_{\text {core }}-1} \varepsilon_{n} I_{\lambda}^{\text {core }}\left(\varepsilon_{n}\right) J_{0}\left(2 \pi f \varepsilon_{n}\right) \\
& \quad+\pi h_{\text {core }} \varepsilon_{\text {in }} I_{\lambda}^{\text {in }} J_{0}\left(2 \pi f \varepsilon_{\text {in }}\right) ;
\end{aligned}
$$




$$
\begin{aligned}
& \text { - } \breve{I}_{\lambda}^{\text {shell }}(f) \approx 2 \pi h_{\text {shell }} \sum_{m=1}^{N_{\text {shell }}-1} \varepsilon_{m} I_{\lambda}^{\text {shell }}\left(\varepsilon_{m}\right) J_{0}\left(2 \pi f \varepsilon_{m}\right) \\
& \quad+\pi h_{\text {shell }} \varepsilon_{\text {in }} I_{\lambda}^{\text {in }} J_{0}\left(2 \pi f \varepsilon_{\text {in }}\right)
\end{aligned}
$$

$N_{\text {core }}$ and $N_{\text {shell }}$ being the numbers of intensity values in the core and in the shell directions, respectively, $h_{\text {core }}=\varepsilon_{\text {in }} / N_{\text {core }}$ and $h_{\text {shell }}=\left(\varepsilon_{\text {out }} \varepsilon_{\text {in }}\right) / N_{\text {shell }}, \varepsilon_{n}=n h_{\text {core }}$ and $\varepsilon_{m}=\varepsilon_{\text {in }}+m h_{\text {shell }}$.

\section{Application to $\pi^{1}$ Gru}

\subsection{General characteristics}

The irregular Srb-type variable $\pi^{1} \mathrm{Gru}$, from 5.4 to 6.7 visual magnitude in 150 days, is one of the brighter $\mathrm{S}$ stars observable from Parañal mountain and having a dusty shell (Knapp et al. 1999), located at a distance of $153 \mathrm{pc}$ (Perryman et al. 1997; van Eck et al. 1998). The S stars are evolved bright giants, cooler than $3500 \mathrm{~K}$ with $10^{2}$ to $10^{4} L_{\odot}$ (Groenewegen \& Jong 1998), showing in their spectra weaker bands of $\mathrm{TiO}$ than for $\mathrm{M}$ stars but stronger bands of $\mathrm{ZrO}$ and sometimes $\mathrm{LaO}$ (Merrill 1952). Traditionally considered as transition objects between M oxygen-rich and C carbon-rich stars on the AGB (Iben \& Renzini 1983), they are classified in 2 categories: the intrinsic Tc-Rich $\mathrm{S}$ stars have a circumstellar envelope. The extrinsic Tc-poor S stars do not show any envelope, which is explained by a different evolutionary scenario involving a present white dwarf companion (Jorissen 1998; Jorissen \& Knapp 1998). The giant star $\pi^{1}$ Gru is known to have a faint and far solar-type G0V companion (apparent visible magnitude of 10.9, angular distance of 2.71" from the primary, not changed since 1900) (Feast 1953), considered as having very little effect on the close circumstellar envelope (Mastrodemos \& Morris 1998; Knapp et al. 1999).

With a dereddened $(V-K)_{0}$ color index of 8.57 (van Eck et al. 2000), $\pi^{1}$ Gru has an effective temperature of $3150 \mathrm{~K}$, deduced from the empirical correlation with the stellar surface brightness applied to $M$ giant stars (van Belle et al. 1999). Since non-Mira S stars are on average $180 \mathrm{~K}$ cooler than the oxygen-rich Miras, which are considered as the closest analogs to the giants stars of spectral class later than M4, for the same $(V-K)_{0}$ color index (van Belle et al. 1997), the temperature of $\pi^{1}$ Gru is adjusted to $2970 \mathrm{~K}$.

At the same time, we can use the other van Belle's relations giving the linear radius as a function of the $(V-K)_{0}$ color index (van Belle et al. 1999), to find that $\pi^{1}$ Gru has a radius of $280 R_{\odot}$. Because non-Mira $\mathrm{S}$ stars on average are $110 R_{\odot}$ larger than oxygen-rich Miras (van Belle et al. 1997), the corrected radius of $\pi^{1}$ Gru can be set to $390 R_{\odot}$ (i.e. 24 mas of angular diameter at $153 \mathrm{pc}$ ), yielding a stellar luminosity of about $10700 L_{\odot}$ and an absolute magnitude of -5.32 .

With a C/O ratio of about 0.8 (Smith \& Lambert 1986), photospheres of S-type stars are rather oxygenated. Concerning $\pi^{1}$ Gru, the conditions are very confused. Since $\pi^{1}$ Gru is classified as "E" (emission silicate) (Volk \& Cohen 1989), we could think that its circumstellar dusty shell is mainly composed of silicates. However, detailed studies of this object, like a color-color diagram (Jorissen \& Knapp 1998), show that its shell emission could also be associated with carbon-rich material (Thomas et al. 1976; Knapp et al. 1999).

\subsection{Measured spectral energy distribution}

Beside ground-based photometric measurements in the infrared (Thomas et al. 1976; van Eck et al. 2000; Smith 2003), $\pi^{1}$ Gru spectra were also taken from space with IRAS in 1987

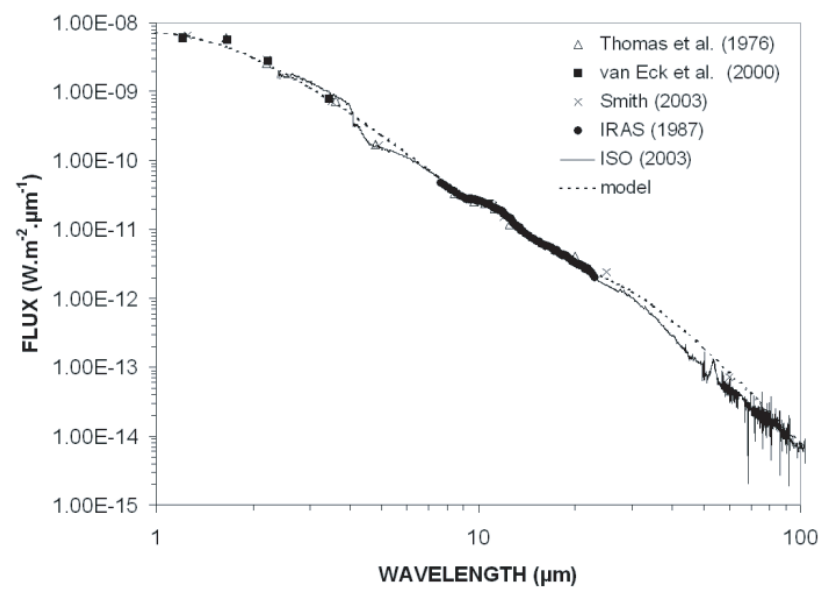

Fig. 10. Optimal fitting model with an effective temperature of the star (dotted line) $T_{\star}=3000 \mathrm{~K}$, overall optical depth at $2.2 \mu \mathrm{m} \tau_{2.2}^{\text {tot }}=0.029$, mixture of $11 \% \mathrm{SiC}$ and $89 \%$ graphite, condensation temperature of the grains $T_{\mathrm{c}}=1500 \mathrm{~K}$, dust density corresponding to a uniform dust flow velocity in $r^{-2}$, and single grain size of $0.05 \mu \mathrm{m}$.

(Joint IRAS Science Working Group 1987) and with ISO in 1996 (Leech et al. 2003; Gry et al. 2003). Figure 10 shows the spectral energy distribution from 1 to $100 \mu \mathrm{m}$, while Fig. 11 shows the plot of a close-up of the dust peak around $11 \mu \mathrm{m}$ that is assumed to be created by a silicone carbide (SiC) shell.

Except for the $K$ magnitude already dereddened by van Eck (van Eck et al. 2000), we corrected the other magnitude measurements for interstellar extinction thanks to the Fitzpatrick's model curve values till the $M$-band (Fitzpatrick 1999) and from the values of Rieke and Lebofsky in the $N$-band (Rieke \& Lebofsky 1985). The van Eck value of $A_{\mathrm{V}}=0.17$ was chosen, so that the ratio of total to selective extinction at $V$, equal to 3.1 on average, allows us to correct the magnitude. At wavelengths longer than $12.5 \mu \mathrm{m}$, the interstellar extinction becomes negligible, compared with the precision of the magnitude. However, the cirrus contamination must be taken into account at $60 \mu \mathrm{m}$ to correct the flux measurement (Egan et al. 1996). While the IRAS fluxes were multiplied by a global "ad-hoc" factor in order to match the $10.5 \mu$ m measurement of Thomas et al., the ISO composite spectrum, composed of disconnected elementary spectra, was reconstructed thanks to "ad-hoc" factors different for each band.

Integration of the linear interpolation of the flux measurements between 0.35 and $100 \mu \mathrm{m}$ found in the literature leads to an observed bolometric flux of $1.13 \times 10^{-8} \mathrm{~W} \mathrm{~m}^{-2}$ At a distance of $153 \mathrm{pc}$, the apparent bolometric magnitude of $\pi^{1} \mathrm{Gru}$ is found to be 0.88 , in relatively good agreement with the van Eck value of 1.07 (van Eck et al. 2000).

\subsection{Fitting the SED with DUSTY}

In order to study the validity of our analytical model without having any visibility measurement, we used the DUSTY code to produce visibility profiles in agreement with the physicochemical parameters listed above and fitting the spectrophotometric data gathered in Figs. 10 and 11. This public domain simulation code solves the problem of radiation transport in a circumstellar dusty environment by analytically integrating the radiative transfer equation (Ivezić \& Elitzur 1996; Ivezić et al. 1999) in planeparallel or spherical geometries. Using the properties of the radiation source and dusty region, it calculates the dust temperature 


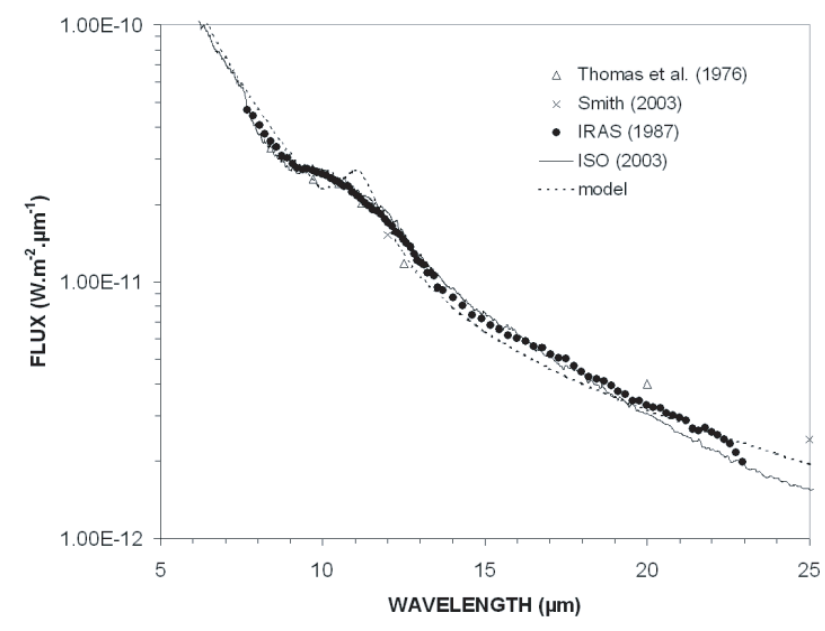

Fig. 11. Plot of the optimal fitting dust features of Fig. 10 between 5 and $25 \mu \mathrm{m}$.

distribution and the radiation field in it. The solution method is based on a self-consistent equation for the radiative energy density, including dust scattering, absorption and emission.

To fit the SED, we first fixed some input DUSTY parameters to values found in the literature:

- an effective temperature of the central star of $3000 \mathrm{~K}$;

- a dust condensation temperature of $1500 \mathrm{~K}$, in good agreement with the sublimation temperature of graphite grains (Goeres 1996);

- a dust density distribution in $r^{-2}$, corresponding to an uniform dust flow velocity;

- a single grain size of $0.05 \mu \mathrm{m}$, so that the dust scattering remains negligible.

Then, we played with the global optical depth and with the chemical composition of the dusty shell. The best fit was obtained with a global optical depth at $2.2 \mu \mathrm{m}$ of about $0.029 \pm$ 0.007 and with a dust grain mixture of $11 \pm 1 \%$ of silicone carbide and $89 \pm 1 \%$ of graphite. Figures 10 and 11 show the final fit obtained. Although the entire spectral energy distribution between 1 and $100 \mu \mathrm{m}$ is strongly constrained by the overall optical depth, the percentage of $\mathrm{SiC}$ was obtained considering that the integrated DUSTY flux between 9 and $13 \mu$ m was approximately equal to the measured integrated flux given by IRAS in the same spectral band.

According to the $\mathrm{S}$ star classification (Jorissen \& Knapp 1998), most of the stars belonging to the $C$ class are surrounded by a dense O-rich circumstellar shell. In our best-fit model, we find that the circumstellar shell of $\pi^{1}$ Gru is optically thin $\left(\tau_{2.2}^{\text {tot }} \approx 0.029\right)$ and contains a majority of carbonaceous grains. In fact, it was showed that, during its variation cycle of 150 days, this star moves in IRAS color-color diagrams from a region composed of stars surrounded by silicate-rich circumstellar envelopes to a region composed of stars surrounded by carbonrich envelopes (van der Veen \& Habing 1988; Jorissen \& Knapp 1998).

\subsection{DUSTY intensity and visibility profiles}

Once the physicochemical features of $\pi^{1}$ Gru determined thanks to the SED, we used the DUSTY code to calculate the radial intensity and visibility profiles.

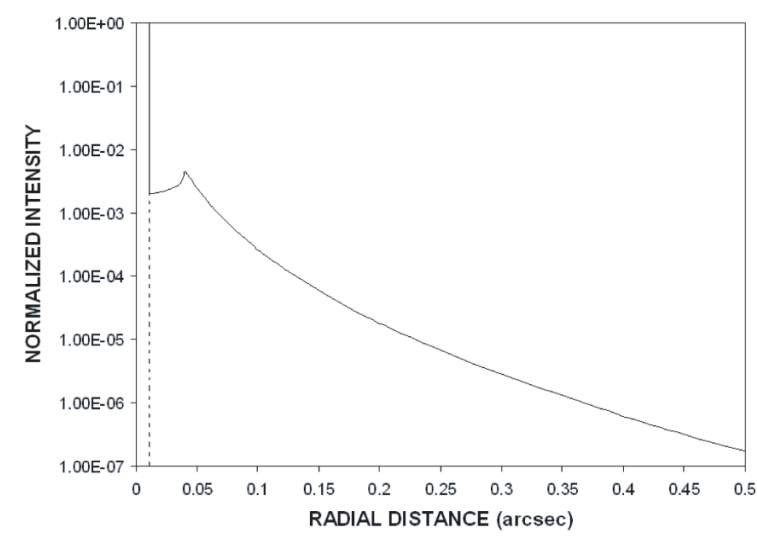

Fig. 12. Central star (dotted line) and DUSTY (full line) normalized intensity profiles obtained with the set of parameters fitting the $\pi^{1}$ Gru's SED measurements.

Figure 12 shows the emergent intensity produced by the DUSTY code, normalized to that of the central star $I_{2.2}^{\star} \approx 1.084 \times$ $10^{7} \mathrm{Jy} \operatorname{arcsec}^{-2}$. By numerical angular integration of the DUSTY intensity profile in the core and shell directions, we find that the envelope flux at $2.2 \mu \mathrm{m}$ is equal to $F_{2.2}^{\text {env }}=\int_{0}^{\infty} 2 \pi \varepsilon I_{2.2}^{\text {env }}(\varepsilon) \mathrm{d} \varepsilon \approx$ $534 \mathrm{Jy}$, which corresponds to about $14.9 \%$ of the stellar flux, given by $F_{2,2}^{\star}=\pi \varepsilon_{\star}^{2} I_{2.2}^{\star} \approx 3585 \mathrm{Jy}$.

A very sharp maximum of intensity appears at the inner boundary with the amplitude of about $49330 \mathrm{Jy} \mathrm{arcsec}^{-2}$, i.e. $0.46 \%$ of the central intensity, creating a discontinuity in the slope of the intensity profile and forming a bright annulus around the central star, confirming that the dusty shell around the star is optically thin (Rowan-Robinson 1980; Lefèvre et al. 1982). The angular distribution of the radiation emitted by the circumstellar medium clearly varies differently in the core and in the shell directions (Mitchell \& Robinson 1978). Figure 12 shows that the angular inner dust radius from DUSTY is located at around 40 mas from the center of the central star. With a ratio between the inner radius boundary and the central star of 3.94, DUSTY foresees that the angular diameter of the central star is around 20.52 mas, not all that far from the value of 24 mas estimated thanks to the $(V-K)_{0}$ color index (van Belle et al. 1999).

Figure 13 shows the DUSTY visibility profile obtained by Hankel transform of the intensity profile, superimposed on visibility profile of the central star alone, considered as an uniform disk, for comparison. Figure 14 shows the incident intensity profile given by the analytical thin-shell model superimposed on the profile given by DUSTY.

Although the analytical thin-dusty-shell model reproduces the global shape of the DUSTY intensity profile fairly well, presenting a sharp maximum of intensity in the direction of the inner boundary, one can notice some slight differences between both profiles. In particular, the intensity profile of the thin-dustyshell model appears flatter than the DUSTY one in the core region, starting from a higher value in the direction of the limb of the star and ending at a lower value in the direction of the inner boundary. In contrast, both profiles tend to converge relatively well in the shell region.

\section{Parameter estimation}

As it is known that ad-hoc geometrical models can provide physical parameters that can be used as inputs for hydrodynamic calculations (Perrin et al. 2004), we also compared our analytical thin-shell model with a simple geometrical model composed of 
Table 1. Best-fitted model parameters and their associated final $\chi^{2}$. Errors on the parameters are given by their confidence interval at $68 \%$.

\begin{tabular}{|c|c|c|c|c|c|c|c|}
\hline Model & param1 & param2 & param3 & param4 & param5 & param6 & $\overline{\chi^{2}}$ \\
\hline & $\begin{array}{c}\varepsilon_{\star} \\
(\mathrm{mas})\end{array}$ & $\begin{array}{l}I_{\text {disk }} / I_{\star} \\
\left(\times 10^{-3}\right)\end{array}$ & $\begin{array}{l}\varepsilon_{\text {disk }} \\
(\text { mas })\end{array}$ & $\begin{array}{l}I_{\text {ring }} / I_{\star} \\
\left(\times 10^{-3}\right)\end{array}$ & $\begin{array}{l}L_{\text {ring }} \\
\text { (mas) }\end{array}$ & $\begin{array}{c}\varepsilon_{\text {ring }} \\
(\text { mas })\end{array}$ & \\
\hline $\begin{array}{l}\text { Geometrical model: } \\
\text { central star + disk + ring }\end{array}$ & $10.25 \pm 0.08$ & $0.23 \pm 0.07$ & $156 \pm 14$ & $2.5 \pm 0.3$ & $49 \pm 17$ & $40 \pm 8$ & 4.58 \\
\hline & $\begin{array}{c}\varepsilon_{\star} \\
(\mathrm{mas})\end{array}$ & $\begin{array}{c}\kappa_{2.2}^{\text {in }} D \\
\left(\mathrm{mas}^{-1}\right)\end{array}$ & $\begin{array}{l}T_{\text {in }} \\
(\mathrm{K})\end{array}$ & $\begin{array}{c}\varepsilon_{\text {in }} \\
(\mathrm{mas})\end{array}$ & $\begin{array}{c}\varepsilon_{\text {out }} \\
(\text { mas })\end{array}$ & & \\
\hline $\begin{array}{l}\text { Analytical thin-shell model } \\
\text { with } T_{\star}=3000 \mathrm{~K}, p=2\end{array}$ & & & & & & & \\
\hline $\begin{array}{l}\text { and } \beta=1 \\
\text { and } \beta=1.5\end{array}$ & $\begin{array}{l}10.23 \pm 0.05 \\
10.23 \pm 0.05\end{array}$ & $\begin{array}{l}0.05 \pm 0.03 \\
0.12 \pm 0.06\end{array}$ & $\begin{array}{l}1024 \pm 66 \\
902 \pm 53\end{array}$ & $\begin{array}{l}46.2 \pm 3.0 \\
46.5 \pm 2.9\end{array}$ & $\begin{array}{l}480 \pm 233 \\
486 \pm 235\end{array}$ & & $\begin{array}{l}1.11 \\
1.04\end{array}$ \\
\hline and $\beta=2$ & $10.23 \pm 0.05$ & $0.29 \pm 0.15$ & $804 \pm 43$ & $46.9 \pm 2.9$ & $493 \pm 238$ & & 0.98 \\
\hline$\beta=1$, and $T_{\text {in }}=1500 \mathrm{~K}$ & $10.22 \pm 0.05$ & $0.005 \pm 0.002$ & - & $37.7 \pm 3.6$ & $186 \pm 31$ & & 3.50 \\
\hline $\begin{array}{l}\beta=1, \text { and } \kappa_{2.2}^{\text {in }} D=5.91 \times 10^{-3} \mathrm{mas}^{-1} \\
\beta=1, \kappa_{2}^{\text {in }} D=5.91 \times 10^{-3} \mathrm{mas}^{-1}\end{array}$ & $10.23 \pm 0.05$ & - & $1371 \pm 124$ & $40.3 \pm 5.2$ & $194 \pm 26$ & & 2.86 \\
\hline and $T_{\mathrm{in}}=1500 \mathrm{~K}$ & $10.22 \pm 0.04$ & - & - & $35.6 \pm 0.6$ & $207 \pm 23$ & & 3.86 \\
\hline DUSTY model & 10.26 & 5.38 & 1500 & 40.4 & $2.02 \times 10^{5}$ & & \\
\hline
\end{tabular}

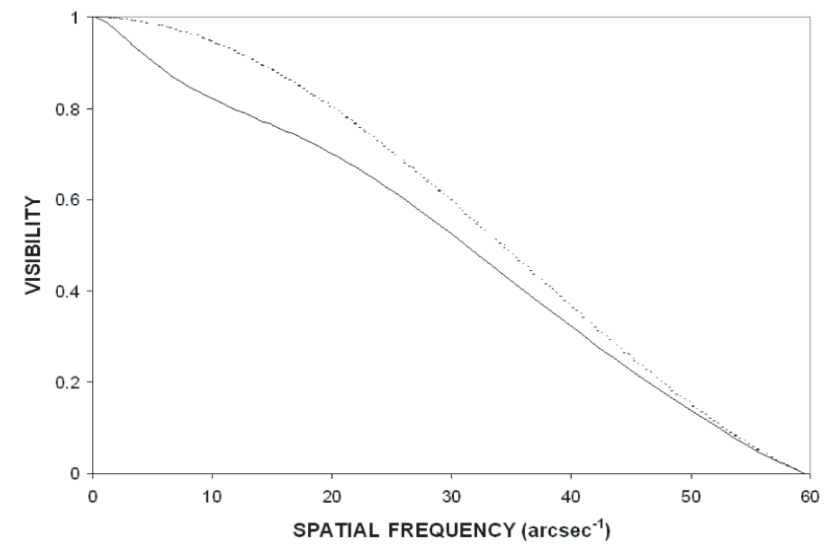

Fig. 13. DUSTY visibility profile (solid line) obtained by Hankel transform of the intensity profile of Fig. 12 superimposed on the visibility profile of the central star alone considered as an uniform disk (dotted line).

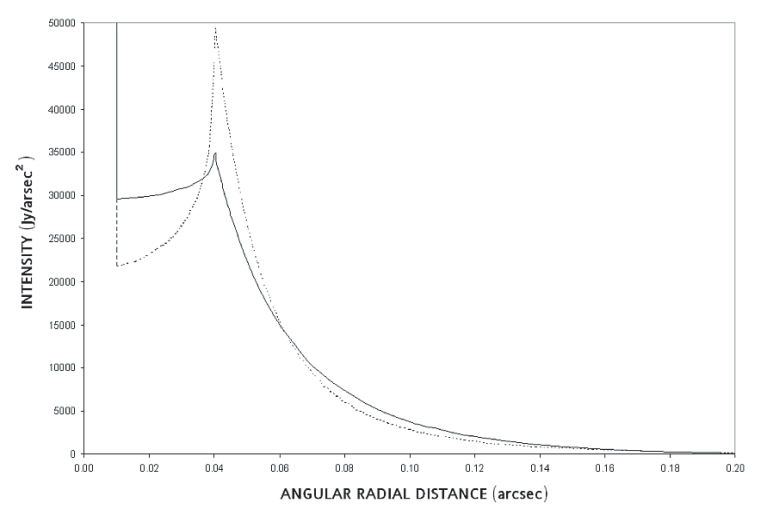

Fig. 14. Analytical thin-shell model (solid line) superimposed on the $\pi^{1}$ Gru's intensity model given by DUSTY (dotted line), obtained with an arbitrary set of input parameters comparable with DUSTY.

a central star considered as an uniform disk surrounded by an envelope described by the sum of a circular uniform disk with a circular uniform ring.
The free independent parameters of the models can be determined thanks to the well-known iterative Levenberg-Marquardt algorithm minimizing the $\chi^{2}$ function defined by

$\chi^{2}\left(a_{l}\right)=\sum_{k=1}^{N_{\text {meas }}} \omega(k)\left[V_{\text {meas }}(k)-V_{\text {model }}\left(f(k) ; a_{l}\right)\right]^{2}$,

where $\omega(k)$ is the weight associated to the visibility measurement $V_{\text {meas }}(k)$, and $V_{\text {model }}\left(f(k) ; a_{l}\right)$ the visibility of the model measured at the spatial frequency $f(k)$ for a given model parameter $a_{l}$. Given the likely range of errors for the VLTI interferometric visibilities $(\approx 0.01)$, the weights associated to the measurements given by the DUSTY code were arbitrarily set to $10^{4}$. The minimization process uses the analytical expression of the first derivatives of the visibility function according to each independent parameter.

We can use this method in order to recover, in particular, the radius of the inner boundary given by DUSTY, which is located at 40.4 mas from the center. Table 1 presents the final parameters values for each model. These parameters must be compared to the corresponding ones used or calculated by the DUSTY code, i.e. $T_{\text {in }}=1500 \mathrm{~K}, \varepsilon_{\text {out }} / \varepsilon_{\text {in }}=5000, \varepsilon_{\star} \approx 10.26$ mas, $\varepsilon_{\text {in }} \approx 40.4 \mathrm{mas}$, and $I_{\text {in }} / I_{\star} \approx 4.55 \times 10^{-3}$. The errors on the final parameters are given by the diagonal elements of the final covariance matrix.

The 6 free parameters of the geometrical model are the angular radius $\varepsilon_{\star}$ of the central star, the relative intensity $I_{\text {disk }} / I_{\star}$ and the angular radius $\varepsilon_{\text {disk }}$ of the circumstellar disk, the relative intensity $I_{\text {ring }} / I_{\star}$, the mean angular radius $\varepsilon_{\text {ring }}$, and the thickness $L_{\text {ring }}$ of the circumstellar ring.

The 5 free parameters of the analytical thin-dusty-shell models are the angular radius $\varepsilon_{\star}$ of the central star, the intensity coefficient $\kappa_{2.2}^{\text {in }} D$, the inner boundary temperature $T_{\text {in }}$, and the inner and outer radii $\varepsilon_{\text {in }}$ and $\varepsilon_{\text {out }}$. Figure 15 shows the visibility differences between DUSTY and the geometrical and analytical thin-dusty-shell models.

\section{Discussion of the models}

\subsection{Central star + uniform disk + uniform ring}

This 6-free-parameter model considers that the shape of the envelope intensity is described by a uniform intensity ring of finite 


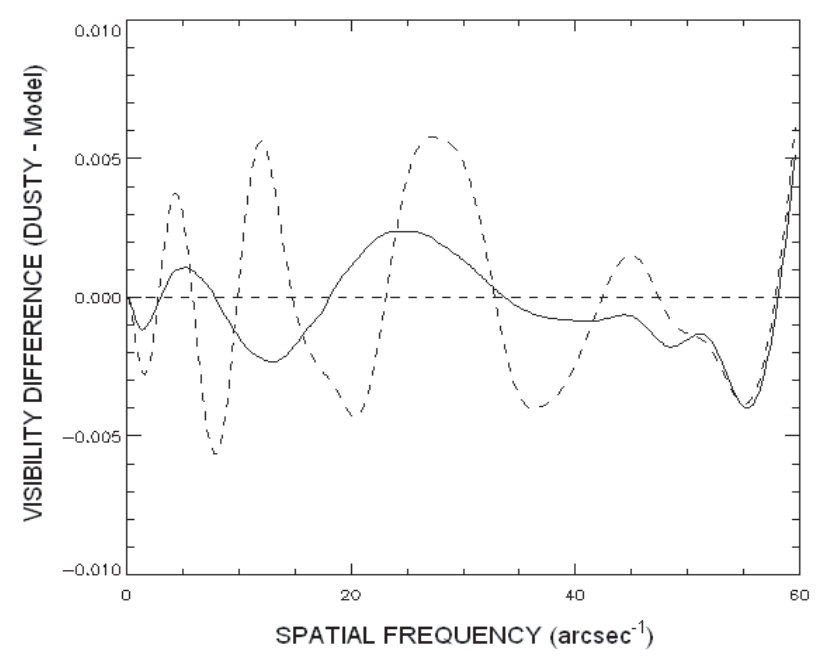

Fig. 15. Visibility difference between DUSTY and the models with the parameters given in Table 1. Solid line: DUSTY - [analytical thin-shell model with $\beta=1$ and 5 free parameters]. Dashed line: DUSTY - [geometrical model].

thickness superimposed on a constant background until an outer angular radius. One can notice that it gives a value of the ring's mean radius (40 mas) that is very close to the DUSTY inner boundary radius (40.4 mas). The envelope flux $F_{2.2}^{\text {env }}$ is found, by angular integration of the envelope intensity profile, to be equal to $14.7 \%$ of the stellar flux, so very close to the value of $14.9 \%$ calculated with DUSTY.

\subsection{Central star + analytical thin-dusty-shell}

This 5-free-parameter model considers that the dusty-shell intensity can be divided into a core and a shell part. The core intensity is proportional to the geometrical thickness, while the shell intensity is proportional to the geometrical thickness multiplied by the function $B_{\lambda}\left(T_{\mathrm{g}}(\varepsilon)\right)$, depending on $\beta$, which describes the wavelength decrease of the optical efficiency $\left(Q_{\lambda} \propto \lambda^{-\beta}\right)$. The resulting shell intensity is close to the DUSTY one, as seen in Fig. 14. This model gives a lower $\chi^{2}$ value than given by the geometrical model with an inner boundary radius of 46.2 to 46.9 mas, not all that far from the DUSTY value of 40.4 mas.

This model also allows us to define the inner boundary grain temperature, which strongly depends on $\beta$. For $\beta=1$, we find the nearest values of the inner boundary temperature $(1024 \mathrm{~K})$ and radius (46.2 mas) to those used or given by DUSTY (1500 K and 40.4 mas). Moreover, we can use numerical angular integration to find that the relative flux $F_{2.2}^{\text {env }} / F_{2.2}^{\star}$ is always near the DUSTY value for the 3 cases $\beta=1(15.01 \%), \beta=1.5(14.92 \%)$, and $\beta=2(15.00 \%)$.

One also notices that the values found for the outer boundary radius are much lower than the value used by DUSTY. This can be explained if we remember that the total monochromatic visibility is related to the fractional contributions of the 2 components $F_{\lambda}^{\star}$ and $F_{\lambda}^{\text {env }}$ to the total flux $F_{\lambda}^{\text {tot }}=F_{\lambda}^{\star}+F_{\lambda}^{\text {env }}$, according to

$V_{\lambda}(f)=f_{\lambda}^{\star} V_{\lambda}^{\star}(f)+f_{\lambda}^{\mathrm{env}} V_{\lambda}^{\mathrm{env}}(f)$,

where $f_{\lambda}^{\star}=F_{\lambda}^{\star} / F_{\lambda}^{\text {tot }}$ and $f_{\lambda}^{\text {env }}=F_{\lambda}^{\text {env }} / F_{\lambda}^{\text {tot }}$. Because the envelope visibilities given by DUSTY and our model are very close to each other, the relative envelope fluxes, obtained by angular integration of the intensity until the outer radius, are nearly identical. As the intensity profile of our model notably differs from DUSTY in the core direction (see Fig. 14), the model outer boundary radius, giving the same envelope flux as DUSTY, must be much smaller than the outer radius of DUSTY.

This result also has a strong influence on the $\kappa_{2.2}^{\text {in }} D$ comparison with DUSTY. According to Eq. (22) and using the values given or used by DUSTY $\left(\varepsilon_{\star} \approx 10.26 \mathrm{mas}, I_{\text {in }} / I_{\star} \approx 4.55 \times 10^{-3}\right.$, $\varepsilon_{\text {in }} \approx 40.4 \mathrm{mas}, T_{\text {in }}=1500 \mathrm{~K}, \varepsilon_{\text {out }} / \varepsilon_{\text {in }}=5000$, and $T_{\star}=$ $3000 \mathrm{~K}$ ), we can calculate that $\kappa_{2.2}^{\text {in }} D \approx 5.38 \mathrm{mas}^{-1}$. If we use this as the starting value of the $\kappa_{2.2}^{\text {in }} D$ parameter of the analytical thindusty-shell model with 5 free parameters, the iterative $\chi^{2}$ minimization process diverges. The reason for this is that the outer boundary radius of $5000 \varepsilon_{\text {in }}$ is clearly incompatible with the analytical thin-dusty-shell model, as explained before. If we use a much smaller value $\varepsilon_{\text {out }} \approx 5 \varepsilon_{\text {in }}$, i.e. $\kappa_{2.2}^{\text {in }} D \approx 5.91 \times 10^{-3} \mathrm{mas}^{-1}$, the process converges towards the values given in Table 1 .

However, we could approximately find again the value of the DUSTY overall optical depth $\tau_{2.2}^{\text {tot }} \approx 0.029$ thanks to Eq. (7) if we consider that the overall optical depth of the thin dusty shell is given by

$\tau_{\lambda}^{\mathrm{tot}}=\tau_{\lambda}\left(r_{\mathrm{out}} ; \varepsilon=0\right) \approx \kappa_{\lambda}^{\mathrm{in}} D\left(\frac{\varepsilon_{\mathrm{in}}}{\varepsilon_{\mathrm{out}}}\right)^{p}\left(\varepsilon_{\mathrm{out}}-\varepsilon_{\mathrm{in}}\right)$.

For example, the 4-free-parameter model with $p=2, \kappa_{\lambda}^{\text {in }} D=$ $5.91 \times 10^{-3} \mathrm{mas}^{-1}, \varepsilon_{\text {in }}=40.3 \pm 5.2 \mathrm{mas}$, and $\varepsilon_{\text {out }}=194 \pm 26 \mathrm{mas}$, gives an overall optical depth at $2.2 \mu \mathrm{m}$ of $0.039 \pm 0.013$.

\section{Interpretation}

The output parameters of each model give information on the constraints to be respected if we want to choose a model that simultaneously describes the morphology and the physics of an astrophysical source surrounded by a thin shell of dust.

\subsection{Fitting the morphology}

The geometrical shape of the model must be close to the object morphology. If this is not respected, 2 drawbacks may appear: a high $\chi^{2}$ value, caused by a poor fit, and non-reliable values for the model parameters, which is more problematic. Contrary to what could be thought, a well-fitting model does not necessary give the most reliable parameters. This is shown in the following section, where we succeed in recovering values of parameters closer to the DUSTY ones, but with a worse $\chi^{2}$ value, in fixing some free parameters.

\subsection{Choosing the free parameters}

The number of free parameters is also a key-element. This is shown in the following example in which we reduce the number of free parameters of the analytical thin-dusty-shell model in replacing some of them by their values given by the DUSTY radiative transfer code fitting the spectrophotometric measurements:

1. Setting the inner boundary temperature to the value of $1500 \mathrm{~K}$ used as an input parameter of the DUSTY code (and again $p=2$ and $\beta=1$ ), leads to the values of the 4 other parameters to $\varepsilon_{\star} \approx 10.22$ mas, $\kappa_{2.2}^{\text {in }} D \approx 5 \times 10^{-3} \mathrm{mas}^{-1}, \varepsilon_{\text {out }} \approx$ 186 mas, and $\varepsilon_{\text {in }} \approx 37.7$ mas, with a final $\chi^{2}$ equal to 3.50 . The value found for the inner boundary radius is lower than the value given with the 5-free-parameter model (46.2 mas) and closer to the one given by the DUSTY code (40.4 mas). 


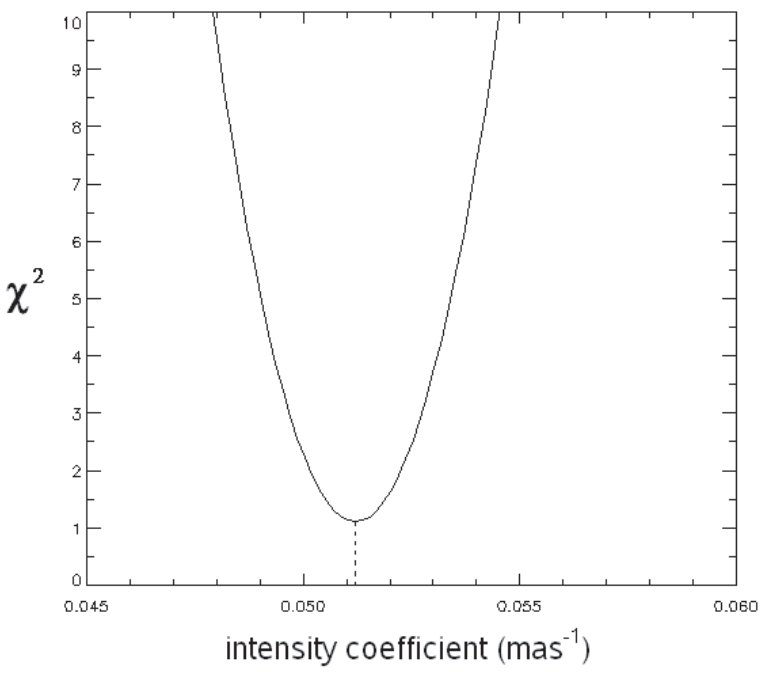

Fig. 16. Variation in the $\chi^{2}$ versus the intensity coefficient $\kappa_{22}^{\text {in }} D$ for the analytical thin-dusty-shell model with 5 free parameters. The dashed vertical line gives the value corresponding to the minimum $\chi^{2}$.

2. Setting the intensity coefficient $\kappa_{2.2}^{\text {in }} D$ to the value of $5.91 \times$ $10^{-3}$ mas $^{-1}$ (and also $p=2$ and $\beta=1$ ) leads to the values of the 4 other parameters to $\varepsilon_{\star} \approx 10.23$ mas, $\varepsilon_{\text {out }} \approx 194$ mas, $\varepsilon_{\text {in }} \approx 40.3 \mathrm{mas}$, and $T_{\text {in }} \approx 1371 \mathrm{~K}$, with a final $\chi^{2}$ equal to 2.86 . In this case, the value found for the inner boundary radius is very close to the one given by DUSTY. The value of the inner boundary temperature is significantly higher than the value given with the 5-free-parameter model (1371 K against $1024 \mathrm{~K}$ ) and closer to the DUSTY one, while the final $\chi^{2}$ is 2.6 times higher.

3. Finally setting both the intensity coefficient to $5.91 \times$ $10^{-3}$ mas $^{-1}$ and the inner boundary temperature to $1500 \mathrm{~K}$ (again with $p=2$ and $\beta=1$ ) leads to the values of the 3 other parameters to $\varepsilon_{\star} \approx 10.22$ mas, $\varepsilon_{\text {out }} \approx 207$ mas, and $\varepsilon_{\text {in }} \approx 35.6$ mas, with a final $\chi^{2}$ equal to 3.86 . The value found for the inner boundary radius now becomes significantly lower than the DUSTY one, while the higher value of the $\chi^{2}$ means a worse visibility fit than before.

This results are a direct consequence of the different behaviors of the $\chi^{2}$ function according to the free parameters of the models. In Figs. 16-18, one can see that the inner boundary temperature is the least constraining parameter of the analytical thin-dusty-shell model. Indeed, the slope of the $\chi^{2}$ function around its minimum is flatter according to the inner boundary temperature compared to the inner boundary radius or to the intensity coefficient. This means that setting the inner boundary temperature to a given a priori value will affect the fitting process less than setting the radius or the intensity coefficient.

From this study, we can conclude that a well-fitting model (in terms of small $\chi^{2}$ value) does not necessarily represent the real physical conditions. Thus, the choice of the model must be driven by 2 basic criteria, namely:

1. The shape of the model that must be deduced from the physics of the object to get a good fitting of the data.

2. The number of free parameters of the model that must be neither too high nor too low, which in both cases could give non-reliable parameter values.

Consequently, if we want to reduce the number of free parameters, we really need to use other observational techniques to get

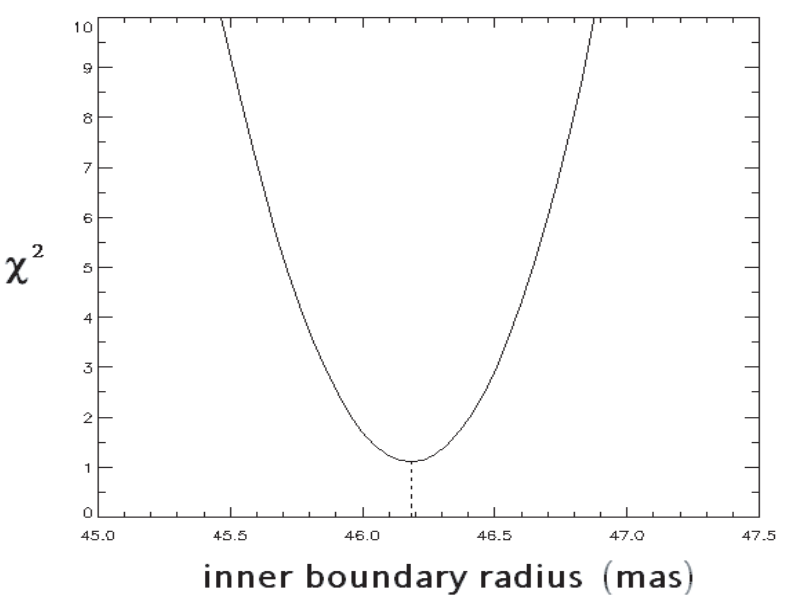

Fig. 17. Variation in the $\chi^{2}$ versus the inner boundary radius for the analytical thin-dusty-shell model with 5 free parameters. The dashed vertical line gives the value corresponding to the minimum $\chi^{2}$.

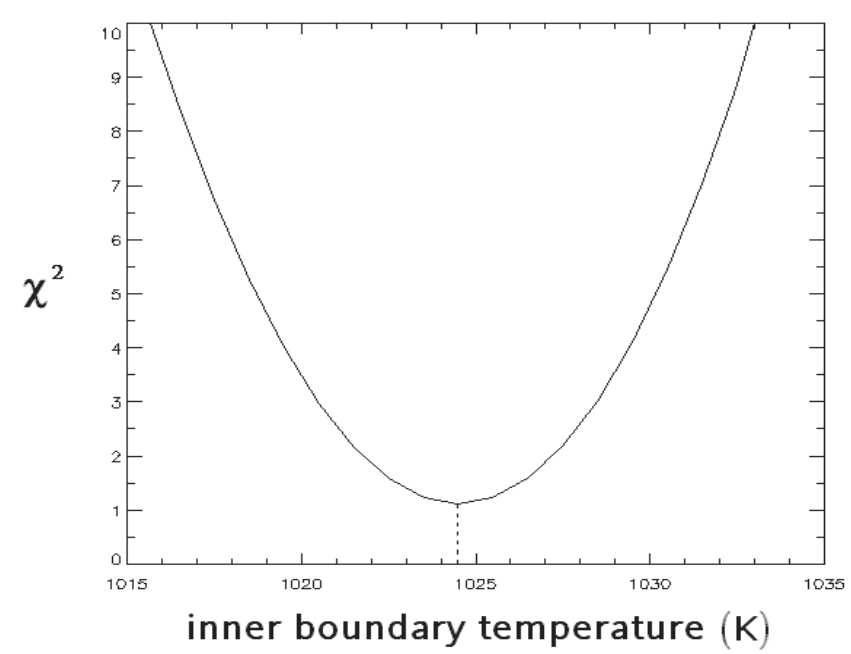

Fig. 18. Variation in the $\chi^{2}$ versus the inner boundary temperature for the analytical thin-dusty-shell model with 5 free parameters. The dashed vertical line gives the value corresponding to the minimum $\chi^{2}$.

a priori knowledge of some parameters or at least get ranges of values. For instance, as we could have seen before, fixing the value of the inner boundary temperature of the analytical thin-shell model gives a reliable value for the inner boundary radius. This temperature could, for example, be estimated by fitting spectrophotometric data. However, rather than fitting the visibility function and the spectral energy distribution separately, leading to 2 different sets of final parameters, the better approach consists in fitting the correlated flux, defined by the product $F_{\lambda} V_{\lambda}$. Particularly suitable for interpreting interferometric polychromatic data, this global approach, which does not reduce the number of free parameters, has the big advantage of simultaneously fitting the SED and the visibility function, giving a unique set of final parameters that is consistent with the 2 sets of measurements (spectrum and visibility). Thus, the combination of various observational techniques should allow us to find model parameters compatible with the interferometric measurements and coherent with the physical conditions. If this is not the case, it might be necessary to use an even smaller number of model parameters, or, more drastically, to use another more suitable model. 


\section{Conclusions}

High resolution interferometry offers the astronomical community high capabilities for determining the geometrical morphology of an object. This observational technique opens a new window toward understanding the very close environment of late-type stars. The main difficulty is still interpreting the data coming from this type of instrument. One solution consists in using analytical models that allow fast calculations before trying to spend more time fitting interferometric data by purely radiative transfer models that rarely give straightforward information. The great advantage of this method is to allow us to automatically determine the values of some physical parameters with their confidence intervals. To decide whether a model is more appropriate than another or not, we can compare its results with those obtained with other observing techniques like spectrophotometry.

In our present paper, we studied its validity for the special case of the interferometric observation of the circumstellar environment of the S-type star $\pi^{1}$ Gru. We proposed a new analytical model of the thin shell of dust deduced from the radiative transfer equation. Although the general form of this model requires a total set of 5 free parameters, we shown that it can be reduced to 4 free parameters with an increase in reliability of the value of the inner boundary radius given by the $\chi^{2}$ minimization of the visibility measurements. For example, the fit of the spectral energy distribution can be used to determine the inner boundary temperature, a parameter poorly constrained by only visibility measurements. However, the use of the correlated flux, defined by the product $F_{\lambda} V_{\lambda}$, allows us to simultaneously fit the SED and the visibility function, giving a set of reliable final parameters without any need of reducing the number of free parameters. To interpret polychromatic visibility measurements with the VLTI focal instruments AMBER and MIDI, we plan to follow this global approach.

Acknowledgements. We want to thank Pr. Jorissen from ULB-IAA (Belgium) for his critical reading of the paper and to have kindly shared his expertise about S-stars. Special thanks must be expressed to Dr. Stee from OCA-Gemini (France) for his constructive suggestions for clarifying the object of the paper. The animated debates we had on many occasions with Prs. Gay and Rabbia from OCAGemini on the general topics of the radiative transfer have greatly helped us to define means to express the analytical thin-dusty-shell model. We also do not want to forget either Dr. Ohnaka from MPIR (Germany) or Dr. Helvaci from AUSF-DASS (Turkey) for the helpful discussions concerning the use of the DUSTY code to fit the spectrophotometric data. Mr. Sacuto benefits from a PHD grant from the Conseil Régional Provence - Alpes - Côte d'Azur (France) managed by ADER-PACA.

\section{References}

Abramowitz, M., \& Stegun, I. A. 1972, Handbook of Mathematical Functions, Ser. 55: Applied Mathematics (National Bureau of Standards)

Barakat, R., Parshall, E., Sandler, B. H., et al. 1998, J. Opt. Soc. Am. A, 15, 652 Bracewell, R. 1999, The Fourier Transform and its Applications (New York: McGraw-Hill)

Danchi, W. C., Bester, M., Degiacomi, C. G., Greenhill, L. J., \& Townes, C. H. 1994, AJ, 107, 1469

Egan, M. P., Kwok, S., Leung, C. M., et al. 1996, A\&A, 308, 738

Feast, M. W. 1953, MNRAS, 113, 510

Fitzpatrick, E. L. 1999, PASP, 111, 63

Goeres, A. 1996, in Hydrogen Deficient Stars, ed. C. S. Jeffery, \& U. Heber, ASP (San Francisco: ASP), 96, 69

Groenewegen, M. A. T., \& Jong, T. D. 1998, A\&A, 337, 797

Gry, C., Swinyard, B., Harwood, A., et al. 2003, ESA SP-1262, Vol. III, The ISO Handbook, LWS - The Long Wavelength Spectrometer (European Space Agency)

Habing, H. J. 1996, A\&AR, 7, 97

Iben, I., \& Renzini, A. 1983, ARA\&A, 21, 271

Ivezić, Z., \& Elitzur, M. 1996, MNRAS, 279, 1011

Ivezić, Z., \& Elitzur, M. 1997, MNRAS, 287, 799

Ivezić, Z., et al. 1999, User Manual for DUSTY, Dept. of Physics and Astronomy, University of Kentucky, Lexington, KY 40506-0055, USA

Joint IRAS Science Working Group 1987, IRAS Low Resolution Spectra (LRS), Tech. Rep. RP-1190, NASA

Jorissen, A. 1998, in on AGB Stars, IAU Symp., 191, 437

Jorissen, A., \& Knapp, G. R. 1998, A\&AS, 129, 363

Knapp, G. R., Young, K., Crosas, M., et al. 1999, A\&A, 346, 175

Leech, K., et al. 2003, ESA SP-1262, Vol. V, The ISO Handbook, SWS - The Short Wavelength Spectrometer (European Space Agency)

Lefèvre, J., Bergeat, J., \& Daniel, J.-Y., 1982, A\&A, 114, 341

Marengo, M. 2000, Ph.D. Thesis, International School for Advanced Studies SISSA-ISAS, Harvard-Smithsonian Center for Astrophysics

Marengo, M., Canil, G., Silvestro, G., et al. 1997, A\&A, 322, 924

Mastrodemos, N., \& Morris, M. 1998, ApJ, 497, 303

Merrill, P. W. 1952, ApJ, 116, 21

Mitchell, R. M., \& Robinson, G. 1978, ApJ, 220, 841

Ohnaka, K., Bergeat, J., Driebe, T., et al. 2005, A\&A, 429, 1057

Papoular, R., \& Pégourié, B. 1986, A\&A, 156, 199

Perrin, G., Ridgway, S. T., Mennesson, B., et al. 2004, A\&A, 426, 279

Perrin, G., Ridgway, S. T., Verhoelst, T., et al. 2005, A\&A, 436, 317

Perryman, M. A. C., Lindegren, L., Kovalevsky, J., et al. 1997, A\&A, 323, L49

Ridgway, S. T., Joyce, R. R., Connors, D., Pipher, J. L., \& Dainty, C. 1986, ApJ, 302,662

Rieke, G. H., \& Lebofsky, M. J. 1985, ApJ, 288, 618

Rowan-Robinson, M. 1980, ApJS, 44, 403

Smith, B. J. 2003, AJ, 126, 935

Smith, V. V., \& Lambert, D. L. 1986, ApJ, 311, 843

Sopka, R. J., Hildebrand, R., Jaffe, D. T., et al. 1985, ApJ, 294, 242

Thomas, J. A., Robinson, G., Hyland, A. R., et al. 1976, MNRAS, 174, 711

van Belle, G. T., Dyck, H. M., Thompson, R. R., et al. 1997, AJ, 114, 2150

van Belle, G. T., Lane, B. F., Thompson, R. R., et al. 1999, AJ, 117, 521

van der Veen, W. E. C. J., \& Habing, H. J. 1988, A\&A, 194, 125

van der Veen, W. E. C. J., Omont, A., Habing, H. J., et al. 1995, A\&A, 295, 445

van Eck, S., Jorissen, A., Udry, S., Mayor, M., \& Pernier, B. 1998, A\&A, 329, 971

van Eck, S., Jorissen, A., Udry, S., et al. 2000, A\&AS, 145, 51

Volk, K., \& Cohen, M. 1989, AJ, 98, 931

Zuckerman, B. 1980, ARA\&A, 18, 263 